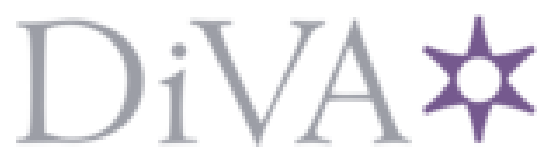

http://www.diva-portal.org

\title{
Postprint
}

This is the accepted version of a paper published in Journal of Pediatric Urology. This paper has been peer-reviewed but does not include the final publisher proof-corrections or journal pagination.

Citation for the original published paper (version of record):

Nevéus, T. (2018)

The amount of urine voided in bed by children with enuresis

Journal of Pediatric Urology

https://doi.org/10.1016/j.jpurol.2018.08.006

Access to the published version may require subscription.

N.B. When citing this work, cite the original published paper.

Permanent link to this version:

http://urn.kb.se/resolve?urn=urn:nbn:se:uu:diva-371522 


\section{THE AMOUNT OF URINE VOIDED IN BED BY CHILDREN WITH}

\section{ENURESIS}

\section{Extended summary}

\section{INTRODUCTION}

Enuretic children wet their beds either because of nocturnal polyuria or nocturnal detrusor overactivity. Polyuric children often respond to desmopressin, whereas children with nocturnal detrusor overactivity are often therapy-resistant and may have low daytime voided volumes. It is logical to assume that the enuretic event in children with nocturnal polyuria occurs with a full bladder, i.e. with the enuretic voided volume (EVV) close to the child's expected bladder capacity (EBC) for his/her age. However, the EVV has only rarely been studied and little is known about how it relates to case history, polyuria or daytime bladder function. The aim of our study was to look at EVV and relate it to voiding chart data and treatment response.

\section{METHODS}

We retrospectively reviewed anamnestic data and voiding charts, including measurement of nocturnal urine production and EVV, in enuretic children with or without concomittant daytime incontinence attending a tertiary center. The EVV was analysed and compared to anamnestic data, voiding chart data and 
response to therapy in accordance with the guidelines of the International Children's Continence Society.

\section{RESULTS}

Data was available for 220 children (age 5-24, median 9), 40 of whom were girls. The prevalence of previous or present daytime incontinence was $42.4 \%$ and $50.5 \%$ sometimes experienced urgency. The average EVV was $54.9 \pm 32.8 \%$ of EBC. EVV correlated highly significantly to nocturnal urine production $(\mathrm{p}<0.001)$. Still, in only 16 children was EVV $\geq$ EBC. EVV was slightly larger in girls $(p=0.013)$ and in children with urgency $(p=0.034)$ but there were no significant EVV differences between children responding or not responding to antidiuretic, anticholinergic or antidepressant therapy. Relevant data are summarized in the Table below.

\section{DISCUSSION}

Case history and daytime voiding chart data give very little information about nocturnal bladder function. The EVV is strongly influenced by nocturnal urine production but only rarely reaches upp to a level close to or exceeding the EBC. The enuretic event only very rarely represents the emptying of a full bladder. Thus, a component of nocturnal detrusor overactivity can be assumed to be present in almost all enuretic children, even children with nocturnal polyuria. The value of the EVV is limited in predicting response to therapy.

Table

Enuresis voided volume (\% of EBC) according to dichotomized variables 


\begin{tabular}{|l|l|l|l|}
\hline & $\mathrm{n} / \mathrm{n}$ & EVV (means $\pm 1 \mathrm{SD})$ & $\mathrm{p}$ value \\
\hline Girl/boy & $34 / 158$ & $67.6 \pm 38.5 / 52.2 \pm 30.9$ & 0.013 \\
\hline Daytime incontinence $(\mathrm{y} / \mathrm{n})$ & $79 / 112$ & $56.1 \pm 38.0 / 53.9 \pm 28.7$ & 0.667 \\
\hline Urgency (y/n) & $89 / 89$ & $60.0 \pm 34.3 / 50.1 \pm 27.1$ & 0.034 \\
\hline Nocturnal polyuria (y/n) & $43 / 144$ & $90.2 \pm 37.6 / 45.6 \pm 22.5$ & $<0.001$ \\
\hline Desmopressin response $(\mathrm{y} / \mathrm{n})$ & $15 / 154$ & $51.3 \pm 23.3 / 56.5 \pm 34.3$ & 0.566 \\
\hline Anticholinergic response (y/n) & $42 / 76$ & $52.0 \pm 36.7 / 56.9 \pm 34.3$ & 0.471 \\
\hline Antidepressant response (y/n) & $36 / 14$ & $54.4 \pm 30.4 / 74.6 \pm 54.6$ & 0.102 \\
\hline
\end{tabular}

Key words: enuresis; voiding chart; voided volumes; nocturnal polyuria; detrusor overactivity

\section{Introduction}

The standard consensus of enuresis pathogenesis is that the child wets his/her bed as a consequence of either nocturnal polyuria or a reduced nocturnal bladder storage capacity, in both cases combined with an inability to adequately awaken from sleep [1]. Children who respond to desmopressin therapy have been shown to usually have nocturnal polyuria [2], whereas desmopressin nonresponders often exhibit nocturnal detrusor overactivity [3]. The recently introduced entity "isolated nocturnal small bladder capacity" probably belongs 
to this latter group, although future studies using overnight cystometry may modify this view [4].

The increased knowledge of enuresis pathogenesis has lead to the recommendation to use voiding charts to assess bladder function and urine production during both day and night in the initial evaluation of the enuretic child [1]. It has then been observed that in addition to nocturnal polyuria, desmopressin responders tend to have normal daytime voided volumes (DVV) $[5,6]$, whereas responders to anticholinergics tend to have low DVV as an indirect sign of detrusor overativity [7]. However, no closer assessment of the prognostic value - if any - of the amount of urine actually voided in bed has been made. This is strange, given that this is the only readily available measure of nocturnal bladder function. I have chosen to call this variable enuresis voided volume (EVV).

Thus, the enuretic bladder is voided either because it is full or it is overactive. In the former case EVV should be similar to the expected bladder capacity (EBC) for the child's age, whereas in the latter case it can almost by definition be assumed to be smaller. It can thus be hypothesized that enuretic children with an EVV near or above the EBC will be desmopressin responders and those with an EVV that is clearly below EBC will be desmopressin nonresponders but might respond to anticholinergics. The present study was undertaken with this hypothesis in mind.

Our aim was to look at the relationship between the EVV and 1) other voiding chart variables, and 2) anamnestic data related to micturition habits or 
treatment response. As a pilot study this might serve as a ground for further, prospective investigations.

\section{Subjects and methods}

This was a retrospective evaluation of children with enuresis attending either one primary care pediatric ward or the tertiary care pediatric nephrology outpatient unit in the same city. All families who managed to complete a voiding chart designed in accordance with ICCS recommendations [8], including nocturnal and daytime voided volumes during one weekend (three nights and two days), were included, provided there was no concomittant neurological, urological or nephrological disorder.

All children were treated in a stepwise fashion according to the ICCS recommendations $[1,9]$ :

1) Exclusion or treatment of concomittant constipation, if present

2) Treatment of concomittant daytime incontinence, if present, using standard urotherapy and, if needed, daytime anticholinergics

3) Treatment with the enuresis alarm or desmopressin (provided constipation and daytime incontinence absent)

4) Treatment with anticholinergics (if both first-line alternatives failed)

5) Treatment with antidepressants

In addition to the treatment listed above all children, at first admission, were given basic bladder advice regarding regular voiding habits and fluid restriction in the evening. All medications were given in full dosage for at least one month. Treatment during steps 1 and 3 above were administered by one general 
pediatrician together with one pediatric nurse, according to written guidelines provided by dr Nevéus. All other treatment (steps 2, 4 and 5) were provided by a pediatric nephrologist (dr Nevéus) supported by a urotherapist. Since this was a retrospective evaluation and no proper clinical trial, response was defined as family satisfaction (i.e. dryness) and not determined from bladder diaries. Noninvasive urodynamics (flowmetry and residual measurements) were performed in all children with daytime incontinence and all with enuresis resistant to first-line therapy.

Anamnestic data, focused on treatment response, daytime voiding habits and the presence or absence of urgency was extracted from patient files. The following voiding chart variables were calculated: daytime micturition frequency, average and maximum DVV without inclusion of the first morning void, EVV and nocturnal urine production during nights with enuresis. The EVV was the increase in diaper weight during the night and the nocturnal urine production was the sum of the EVV and the first morning void. All variables except micturition frequency were transformed into percentages of EBC according to the Hjälmås formula [10], i.e. (age + 1) * $30 \mathrm{~mL}$. The reason for exclusion of the first morning void when calculating DVV was that after dry nights this micturition usually represents the largest volume, but only a minority of the children included could document any dry nights. All voiding charts were completed while the child was free from antienuretic therapy. 
Since the completion of voiding charts is part of the internationally recommended standard evaluation of enuretic children $[1,9]$ no Ethics Review Board permission was sought for this study.

The central outcome variable was the EVV. We looked for significant correlations between this and other scale variables and compared EVV of dichotomized subgroups - such as children with and without urgency and responders and nonresponders to therapies given - using t-tests or nonparametric alternatives. The statistical significance level was set at 95\% ( $p<0.05)$. The Statistics Package for the Social Sciences (SPSS) version 24 was used.

\section{Results}

All in all, 220 children were included. Their ages were $9.6 \pm 3.0$ years (range 524, median 9) and $40(18.2 \%)$ of them were girls. Only 45 (20.4\%) of the children were recruited from primary care. According to the patient files $21.8 \%$ of the patients experienced urgency symptoms, whereas $42.4 \%$ had a history of previous $(21.8 \%)$ or present $(20.6 \%)$ daytime urge incontinence. Their response to antienuretic pharmacological treatment is summarized in Table 1 and their voiding chart data are described in Table 2. Seven children had secondary enuresis, i.e. they had previously experienced dry periods of at least 6 months (usually after alarm therapy). They did not differ from the rest of the children in any of the evaluated parameters (data not shown). None of the children became dry just by the initial basic bladder advice. No pathology was found on the noninvasive urodynamics. 
Sadly, the data regarding response to the enuresis alarm was very incomplete, since the families of successfully treated children tended to fail to report this to the physician and in case of nonresponse it was difficult to determine whether this was due to non-adherence or technical problems.

[Tables 1 and 2 here]

As can be understood from the table, the vast majority of the children had an EVV much below their EBC. In fact, only sixteen (8.3\%) out of the 192 children for whom EVV was available emptied a full bladder at the time of enuresis, i.e. had an EVV $\geq$ EBC.

EVV was slightly higher for girls than boys $(67.6 \pm 38.5 \%$ vs $52.2 \pm 30.9 \%$, $\mathrm{p}=0.013$ ). Somewhat surprisingly, EVV did not differ between children with and without a history of daytime incontinence $(56.1 \pm 38.0 \%$ vs $53.9 \pm 28.7 \%$, $\mathrm{p}=0.67)$ and the value was significantly higher in children with urgency than those without this symptom $(60.0 \pm 34.3 \%$ vs $50.1 \pm 27.1 \%, \mathrm{p}=0.034)$. This discrepancy is explained by the observation that children with previous incontinence turned out to have lower EVV $(44.1 \pm 27 . \%)$ than both those with daytime incontinence at the time of data collection $(67.1 \pm 43.1 \%)$ and those who had never suffered from daytime incontinence $(53.9 \pm 28.7 \%$, one way ANOVA $F(2,188)=5.15 ; p=0.007)$

Response to neither of the three pharmacological therapies was associated with significant differences in EVV (desmopressin responders $51.3 \pm 23.3 \%$ vs nonresponders $56.5 \pm 34.3 \%$, p=0.57; anticholinergic responders $52.0 \pm 36.7 \%$ 
vs nonresponders $56.9 \pm 34.3 \%$, $\mathrm{p}=0.47$; antidepressant responders $54.4 \pm$ $30.4 \%$ vs nonresponders $74.6 \pm 54.6 \%, \mathrm{p}=0.10$ ).

EVV was strongly correlated with nocturnal urine production (Pearson correlation coefficient $0.744, \mathrm{p}<0.001$ ), as illustrated in Figure 1 , and weakly, although significantly, correlated to DVV. The correlation coefficient and p-value for the average voided volume was 0.149 and 0.041 , whereas the corresponding figures for maximum voided volumes was 0.160 and 0.028 . There was no statistically significant correlation between EVV and daytime micturition frequency.

[Figure 1 here]

\section{Discussion}

We looked at EVV, defined as the measured amount of urine lost in bed during wet nights, in children with enuresis and found this variable to be strongly correlated to nocturnal urine production and weakly but significantly correlated to DVV, but that less than ten percent of the children emptied a full bladder.

Girls were found to have slightly larger EVV than boys and, surprisingly, daytime urgency, as well as present but not previous daytime urge incontinence, was found to be significantly associated with increased EVV. Antienuretic therapy response was not reflected by any differences in EVV. 
In a small number of other studies some attention has been given to this variable, usually when focusing on something else. Hansen et al examined mainly desmopressin nonresponders and found EVV not to be related to daytime voided volumes [11]. Kawauchi et al found EVV during alarm therapy to be consistently lower than maximum daytime voided volumes [12]. Hagstroem examined enuretic children during an inpatient fluid provokation test and noted that more than half of the resulting enuresis episodes constituted emptying of a less than full bladder [13], whereas Kim et al found no correlation between DVV and EVV [14]. Finally, the Aarhus group found that a low EVV is related to a low nocturnal urine output [4]. The results from these studies are difficult to extrapolate to enuretic children at large either because of the nature of patient selection [11] or due to the children being woken at night, which may have interfered with nocturnal urine production $[12,14]$. Still, the results are largely in line with our findings.

There are two obvious problems with the EVV variable: some children may have voided several times per night and some may have emptied incompletely, leaving residual urine in the bladder. The former leads to overestimation of EVV, whereas the latter leads to the opposite error.

That children with enuresis may void several times per night is a common observation by parents but very little is known about how prevalent this phenomenon is and whether it is peculiar for a specific pathogenetic subgroup. Furthermore, to answer this question the sleep of the children is usually disturbed, which may influence both sleep, arousal and bladder function during the remainder of the night. In a Danish study on eightteen desmopressin 
nonresponders using the enuresis alarm it was found that seventeen of them sometimes voided more than once per night [15]. In another study on 40 enuretic children with unknown desmopressin response who had an overnight volume sensor applied to the abdomen and were awoken at a predetermined bladder filling 29 were found to have multiple nocturnal micturitions [16]. Anyway, we may assume that multiple enuretic episodes per night should be much more common in children with nocturnal detrusor overactivity than those with nocturnal polyuria, provided the polyuria is not extreme.

Incomplete enuretic voidings have been observed by several authors, but results are conflicting. Provoked nocturnal voidings in normally nonenuretic children are often incomplete [17], as are - not surprisingly - voidings during alarm therapy [15]. But even without an alarm interrupting the micturition as much as half of the enuretic episodes represented incomplete voidings in one study utilizing electromyographic detection of sphincter contractions [18]. On the other hand, Nørgaard, in his landmark studies incorporating ambulatory cystometry, found the enuretic episodes to represent normal, complete bladder emptying [19]. Incomplete enuretic bladder emptying can be assumed to be related to arousal rather than detrusor function or nocturnal urine production. It has been found to be equally common regardless of nocturnal polyuria [18].

The only way to overcome the problems of detecting multiple or incomplete enuretic episodes without disturbing the patient would be continuous overnight assessment of bladder volume using body-worn ultrasonographic probes. In as yet unpublished studies using this methodology at the tertiary care pediatric clinic at Linköping University twelve out of 27 enuretic children were found to void more than once per night whereas only four 
voided incompletely, two of whom (the only ones with large residual urine volumes) also had multiple voids with small EVV (Mattsson S, personal communication).

If we try to take both these sources of error into account one conservative interpretation of our findings would be that the majority of therapy-resistant enuretic children void with less than full bladders even though a small number may interrupt their nocturnal micturitions during the voiding phase. In either case the enuretic episode can not be considered a "normal voiding" occuring at an undesired location and time, as was the view when the old definitions were laid down [20]. A further speculation may be that perhaps multiple enuretic episodes underlie our finding that present urgency as well as present but not previous daytime incontinence is associated with an increased EVV.

We only registered 1-3 nights per patient (although the voiding chart comprised three nights not all children wet their beds during all three nights). It is known that urine production varies between nights [21]. Thus, had we asked the families to record more nights we may have found more differences. On the other hand, fewer families would have been able to complete the voiding charts, especially since we wanted to compare daytime and nighttime bladder function, and the documentation of DVV during school days is usually not feasible.

It should be noted that this was a retrospective assessment of voiding chart data, not an evaluation of treatment efficacy. The data on therapy response is much too patchy and the treatment strategy too individualised for such analyses to be meaningful. Also, the patient sample was obviously biased, most of the children 
being resistant to first-line therapy. Had we included more desmopressin responders I suspect that we would have seen significantly larger EVV values in those children. This subgroup, however, is not the problem: the value of the voiding chart in predicting desmopressin response is already well established [2]. What is remarkable is that we did not find any differences in EVV according to anticholinergic response.

A central finding in this study is that the nocturnal voiding of the enuretic child almost never represents the emptying of a full bladder, not even in children with nocturnal polyuria. This probably means that some degree of detrusor overactivity is common to all, or almost all, enuretic children.

The recent study by Borg et al is highly relevant in this context. They (perhaps wisely) avoided discussing EVV but noted that many enuretic children, although having no symptoms of detrusor overactivity and often responding to desmopressin, had a nocturnal urine production on wet nights lower than their EBC [4].

Thus, we should not see nocturnal polyuria and detrusor overactivity as all-or-nothing phenomena that are either present or absent in the individual child, but mechanisms that are present in various degrees - and when the sum of these factors reach a certain threshold level enuresis occurs. This way of thinking also makes it easier to account for the mismatch between enuresis genotype and phenotype (i.e. enuresis subtype) [22].

The observation that urgency was not related to smaller but greater EVV is also interesting. Lacking cystometry, urgency is the only marker we have to assume 
that detrusor overactivity is present. Maybe our results were to a large extent influenced by children with detrusor overactivity voiding several times per night. Or maybe daytime voiding habits and symptoms do not say much about nocturnal bladder function.

We believe that the EVV, as the only readily available measure of nocturnal bladder function, should be further researched, preferably using methods, such as real-time ultrasound, that without interrupting the sleep of the child makes it possible to detect both multiple and incomplete voidings.

\section{CONCLUSIONS}

We found the amount of urine voided in bed in enuretic children to be highly correlated to nocturnal urine production but only rarely large enough to represent the voiding of a full bladder. Most micturitions while asleep can be assumed to be either incomplete or represent nocturnal detrusor overactivity. It may be speculated that most enuretic children, even those with nocturnal polyuria, also have a degree of nocturnal detrusor overactivity.

\section{Funding}

This research did not receive any specific grant from funding agencies in the public, commercial, or not-for-profit sectors.

\section{Ethical approval}


Since the completion of voiding charts is part of the internationally recommended standard evaluation of enuretic children no Ethics Review Board permission was sought for this study.

\section{References}

[1] Nevéus T, Eggert P, Evans J, Macedo A, Rittig S, Tekgül S, et al. Evaluation and treatment of monosymptomatic enuresis - a standardisation document from the International Children's Continence Society (ICCS). J Urol. 2010;183:441-7.

[2] Rittig S, Schaumburg H, Schmidt F, Hunsballe JM, Hansen AF, Kirk J, et al. Long-term home studies of water balance in patients with nocturnal enuresis. Scand J Urol Nephrol. 1997;31(Suppl 183):25-7.

[3] Yeung CK, Chiu HN, Sit FK. Bladder dysfunction in children with refractory monosymptomatic primary nocturnal enuresis. J Urol. 1999;162:1049-55.

[4] Borg B, Kamperis K, Olsen LH, Rittig S. Evidence of reduced bladder capacity during nighttime in children with monosymptomatic nocturnal enuresis. J Pediatr Urol. 2018;14(2):160.e1-6.

[5] Nevéus T, Tuvemo T, Läckgren G, Stenberg A. Bladder capacity and renal concentrating ability in enuresis - pathogenic implications. J Urol. 2001;165(6 pt 1):2022-5. 
[6] Okur M, Ozen SF, Kocabay K, Cam K, Ozkan A, Uzun H. The effect of maximum voided volume on response to desmopressin therapy in children with enuresis. J Nippon Med Sch. 2012;79:255-8.

[7] Nevéus T. Oxybutynin, desmopressin and enuresis. J Urol. 2001;166(6):2459-62.

[8] Austin P, Bauer S, Bower W, Chase J, Franco I, Hoebeke P, et al. The standardization of terminology of lower urinary tract function in children and adolescents: update report from the standardization committee of the International Children's Continence Society. J Urol. 2014;191(6):1863-5.

[9] Franco I, von Gontard A, De Gennaro M. Evaluation and treatment of nonmonosymptomatic nocturnal enuresis: A standardization document from the International Children's Continence Society. J Pediatr Urol. 2012;9(2):234-43.

[10] Hjälmås K. Micturition in infants and children with normal lower urinary tract. Scand J Urol Nephrol. 1976(Suppl 37):1-106.

[11] Hansen AF, Jørgensen TM. A possible explanation of wet and dry nights in enuretic children. Br J Urol. 1997;80(5):809-11. 
[12] Kawauchi A, Tanaka Y, Naito Y, Yamao Y, Ukimura O, Yoneda K, et al. Bladder capacity at the time of enuresis. Urology. 2003;61(5):1016-8.

[13] Hagstroem S, Kamperis K, Rittig S, Djurhuus JC. Bladder reservoir function in children with monosymptomatic nocturnal enuresis and healthy controls. J Urol. 2006;176(2):759-63

[14] Kim JM, Park JW, Lee CS. Evaluation of nocturnal bladder capacity and nocturnal urine volume in nocturnal enuresis. J Pediatr Urol. 2014;10(3):559-63.

[15] Oredsson AF, Jørgensen TM. Changes in nocturnal bladder capacity during treatment with the bell and pad for monosymptomatic nocturnal enuresis. J Urol. 1998;160(1):166-9

[16] Pretlow RA. Treatment of nocturnal enuresis with an ultrasound bladder volume controlled alarm device. J Urol. 1999;162(3 Pt 2):1224-8

[17] Rasmussen PV, Kirk J, Rittig S, Djurhuus JC. The enuretic episode - a complete micturition from a bladder with normal capacity? A critical reappraisal of the definition. Scand J Urol Nephrol. 1997;31(Suppl 183):23-4 
[18] Hagstroem S, Kamperis K, Rittig S, Rijkhoff NJ, Djurhuus JC. Monosymptomatic nocturnal enuresis is associated with abnormal nocturnal bladder emptying. J Urol. 2004;171(6 Pt 2):2562-6

[19] Nørgaard JP, Hansen JH, Nielsen JB, Rittig S, Djurhuus JC. Nocturnal studies in enuretics. A polygraphic study of sleep-EEG and bladder activity. Scand J Urol Nephrol. 1989;125:73-8

[20] Nørgaard JP, van Gool JD, Hjälmås K, Djurhuus JC, Hellström A-L. Standardization and definitions in lower urinary tract dysfunction in children. Br J Urol. 1998;81(Suppl 3):1-16

[21] Hansen MN, Rittig S, Siggaard C, Kamperis K, Hvistendahl G, Schaumburg HL, et al. Intra-individual variability in nighttime urine production and functional bladder capacity estimated by home recordings in patients with nocturnal enuresis. J Urol. 2001;166(6):2452-5

[22] von Gontard A, Eiberg H, Hollmann E, Rittig S, Lehmkuhl G. Molecular genetics of nocturnal enuresis: clinical and genetic heterogeneity. Acta Paediatr. 1998;87(5):571-8. 


\section{Tables}

TABLE 1

Response to antienuretic therapy.

Drug $\mathrm{n}$ receiving $\mathrm{n}$ responding proportion responding

\begin{tabular}{lccc}
\hline Desmopressin & 180 & 19 & $10.0 \%$ \\
Anticholinergics & 134 & 50 & $37.3 \%$ \\
Antidepressants & 60 & 42 & $70.0 \%$
\end{tabular}

TABLE 2

Voiding chart data. All variables except micturition frequency expressed as percentages of expected bladder capacity for age.

Variable

$\mathrm{n} \quad$ median (range) mean $\pm 1 \mathrm{SD}$

Enuresis voided volume

$19250(1-192) \quad 54.9 \pm 32.8$

Urine production during wet nights

$19397(38-288)$

$107.1 \pm 47.7$

Daytime micturition frequency

$216 \quad 5.5(1.5-15) \quad 5.9 \pm 1.9$

Average daytime voided volume

$21538(15-97)$

$39.8 \pm 14.8$

Maximum daytime voided volume

$216 \quad 63(25-167)$

$66.7 \pm 25.4$ 


\section{Figure legends}

\section{Figure 1}

Enuresis voided volume and nocturnal urine production during wet nights, both variables expressed as percentages of expected bladder capacity for age (Pearson correlation coefficient 0.744, $\mathrm{p}<0.001$ ). 